\title{
MITIGATING COMPANY ADOPTION BARRIERS OF DESIGN- DRIVEN INNOVATION WITH HUMAN CENTERED DESIGN
}

\author{
Baha, Ehsan $(1,2,3)$; \\ Ghei, Taresh (4); \\ Kranzbuhler, Anne (1) \\ 1: Delft University of Technology; \\ 2: University of Montreal; \\ 3: Meaningwise; \\ 4: Philips Consumer Lifestyle
}

\begin{abstract}
In Design-Driven Innovation (D-DI) the meaning of a product or service is radically innovated to introduce a new paradigm that ideally can benefit people, companies, and society as a whole. However, due to the associated risks, most companies are hesitant to engage with and adopt D-DI. Human Centered Design (HCD) is preferred while innovation is limited to incremental change. This dichotomy is also reflected in design literature where D-DI is pitted against HCD. We propose the symbiosis of the two approaches as a strategy to create space for and the adoption of D-DI within companies. An instrumental design case study explores a design-driven service innovation and its adoption in a renowned airline. Results show an adopted D-DI where HCD evidence mitigates for the market and organization uncertainty while D-DI enabled a paradigm shift in the company's current service operation. Advantages and limitations of this mitigation strategy are discussed. With this design precedent, we aim to encourage designers and companies to further explore the benefits of a symbiotic use of D-DI and HCD.
\end{abstract}

Keywords: Service design, Design-driven innovation, Case study, Design process, Human Centered Design

\section{Contact:}

Baha, Ehsan

University of Montreal

School of Industrial Design

Canada

ehsan.baha@umontreal.ca

Cite this article: Baha, E., Ghei, T., Kranzbuhler, A. (2021) 'Mitigating Company Adoption Barriers of Design-Driven Innovation with Human Centered Design', in Proceedings of the International Conference on Engineering Design (ICED21), Gothenburg, Sweden, 16-20 August 2021. DOI:10.1017/pds.2021.471 


\section{INTRODUCTION}

Norman and Verganti (2014), distinguish, represent, and 'pit' two schools of thought regarding innovation design: Human Centered Design (HCD) and Design-Driven Innovation (D-DI). Fundamentally rooted in design, etymologically understood as making sense of things (Krippendorff, 1989), D-DI is an innovation approach where the meaning of a product or service is radically changed. Meaning here is defined as "the 'why' of a product - the profound psychological and cultural reasons people use a product" (Verganti, 2011, p. 384). A change in meaning is akin to a change in purpose where the intent is on creating a new need or experience, rather than a new solution to an existing need (Öberg \& Verganti, 2014). This innovation approach is referred to by Norman and Verganti as 'hill finding' - discovering new hills and parallels Kim and Mauborgne's (2005) 'blue ocean strategy'. A blue ocean personifies an unknown market environment free of competition, where innovations create new demand, and there is ample space for companies to grow. Take video games for example, when Sony PlayStation and Microsoft XBox offered technologically advanced consoles, Nintendo took a different path by focusing on democratizing the gaming experience by deviating from this norm. This shift in gaming experience changed the meaning from individual expert focused to games for everyone, where families could play together without a high degree of expertise as a requirement. D-DI as a radical meaning change is sometimes also combined with Technology Push Innovation (TPI), a radical technology change.

In contrast, HCD is propagated as useful for incrementally improving a product or service meaning, thereby increasing its performance and desirability for people. Also known as Market Pull Innovation, Norman and Verganti refer to this innovation approach as 'hill climbing' - where one makes tiny movements on a hill to slowly discover a path that leads to an increase in height. This process repeats until one is satisfied that they have reached the top of the hill, practiced in what Kim and Mauborgn (2005) call the 'red ocean' after which a 'blue ocean' is needed for company growth free of competition. For example, the successive evolution of the gaming handsets of both Sony PlayStation and Microsoft Xbox: Starting with the first generation of handsets, close attention was given to gamer feedback to iteratively improve the ergonomics, tactile and handling experience of the handsets. New technologies and components like vibration motors for simulating tactile impacts, Bluetooth connectivity for wireless gaming, gesture controls were slowly incorporated into these handsets to give players greater freedom of play and enhanced control over their gaming experience.

The theoretical distinction of D-DI and HCD is also observable in practice where they are applied in dichotomy by companies. Between the two approaches, HCD is generally preferred due to the low associated risks in terms of company and market uncertainty. Consequently, the HCD approach is more established compared to D-DI, which has higher associated acceptance risks and market uncertainty. Although pitting D-DI and HCD for their prominent capabilities makes sense, both of these approaches are considered a necessity for companies. As Norman and Verganti (2014) state:

"The bottom line is that both forms of innovation are necessary. Radical innovation brings new domains and new paradigms, and it creates a potential for major changes. Incremental innovation is how the value of that potential is captured. Without radical innovation, incremental innovation reaches a limit. Without incremental innovation, the potential enabled by radical change is not captured" (p. 82).

Although the value and relationship between these two approaches are advocated for companies, literature and best practices of their successful application by one and the same company is scarce. Case studies are often post-hoc and based upon either one of the two approaches by one company.

An exception to the former is Verganti's (2016) later work where a series of D-DI case studies in companies is bundled. Focusing only on D-DI, the case studies demonstrate D-DI activities and workshops that facilitate collaboration among company staff members with experts and interpreters outside of the company, involved to address the D-DI uncertainties and related risks. Verganti's process of innovation of meaning is based on two principles: 'inside-out' and 'critique'. The inside-out principle regards innovation envisioned by a designer or a company for customers as 'what the designer loves the customers to love', not to be mistaken for simply 'what the designer loves' or 'what the customers love'. Criticism is a practice by peers, experts, and interpreters, emphasizing the differences between diverse perspectives to find a deeper new interpretation. Verganti's process of innovation of meaning precedes a vision. That is, a radical innovation of meaning created individually and possibly based on a HCD approach using methods that help in gathering evidence and research 
useful for creating the vision. For example, people research or context analysis. Acknowledging this possibility, Verganti regards HCD as an established approach, hence does not explore its relationship with D-DI any further than pitting.

Another perspective than pitting to explore the relationship between HCD and DD-I is through their symbiosis. Baha et al. (2012) for example, propose a combination of existing and new meanings as a way to mitigate D-DI uncertainty and related risks. Their work is however limited to the external risks or the market uncertainty of a DD-I. Internal risks or the company uncertainty of a DD-I are not discussed as such. Inspired by and building on the work of Baha et al., we propose the symbiosis of DDI and HCD as a mitigation strategy to create space for and the adoption of D-DI within companies. This premise is explored by means of an instrumental design case study within a renowned airline company.

The remainder of this paper is structured in five sections. We start by reviewing background literature describing the adoption barriers of D-DI, followed by the potential of HCD for mitigating these barriers (Section 2). We then describe a six-month instrumental design case study within a renowned airline as our research context and a reflective practice rooted in research through design as our research approach (Section 3). Next, we present and reflect upon a design-driven service innovation vision as the outcome, its design process, and adoption journey (Section 4). The paper ends with a discussion of three contributions and related limitations (Section 5).

\section{BACKGROUND}

\subsection{Company adoption risks of design-driven innovation}

D-DI is considered a form of radical innovation where the associated meanings of products and services are radically changed (Verganti, 2009). Going beyond just function, this approach also includes the emotional or symbolic values that people might associate towards products (Dell'Era \& Verganti, 2007; Verganti, 2009). Dahlin and Behrens (2005, p.725) suggest three criteria to label an innovation as radical:

Criterion 1: The invention must be novel - i.e. dissimilar from prior inventions.

Criterion 2: The invention must be unique - i.e. dissimilar from current inventions.

Criterion 3: The invention must be adopted - i.e. influence the content of future inventions.

The first two criteria deal with the nature of the innovation while the last criterion determines the successful adoption and diffusion of the innovation in the market by companies as well as individuals. Rogers (1995) defines 'adoption' as the physical acquisition and purchase of the innovation. While Fichman (2000) defines 'diffusion' as a process by which a technology spreads across a population of organizations. While Rodgers and Fichman distinguish, Sandberg and Aarikka-Stenroos (2014, p. 1295) merge adoption and diffusion through their broad definition of radical innovation as "an innovation that creates considerable changes in a firm and/or in a market". For the sake of clarity we subscribe to this entanglement by only using the term adoption, meaning not only the market environmental factors but also the company ethos.

According to Norman and Verganti (2014), radical innovations do not always live up to their proposed expectations. Sandberg and Aarikka-Stenroos' (2014) systematic literature review specifies some of the company barriers to radical innovation adoption. Large established firms are usually concerned about risks and uncertainty relating to feasibility, customer acceptance/resistance (Dooley \& O'Sullivan, 2001; Lai et al., 1993; Singh, 2013), commercial success/failure, and costs as the main adoption barriers. At the same time, these companies face adoption barriers such as restrictive mindsets, organizational inertia, and structured routines (Von Stamm, 1998). Restrictive mindset which is often characterized by being resistant to change, inability to accept new ideas, and a lack of risk-taking is considered as most significant. In particular, De Goey et al. (2016) indicate miscommunication and misunderstanding of certain activities, conflicting views on the development and management process, and disharmony with company culture. Barriers do not only exist for radical innovations. Companies engaged in incremental innovations also face similar barriers. However, the extent and severity of these barriers is higher for radical innovations than for incremental innovations (Sandberg \& Aarikka-Stenroos, 2014). 


\subsection{The advantages and limitations of human centered design}

The Human Centered Design (HCD) approach for any product development is characterised by the immersion, observations, deep analysis of peoples' needs, prototyping, and testing (Brown, 2008, 2009; Liedtka \& Ogilvie, 2011). Designers and researchers spend time in the field observing people, their environments, lifestyles and cultures to get a comprehensive understanding of their needs and problem areas. Consequently, HCD is heavily focused on people, their needs and experiences. HCD methods are also used extensively to test for and validate solutions, with each cycle of rapid prototyping and testing leading to more complete mature prototypes. However, HCD's focus on people needs tends to restrict the solution space to the paradigm that the people are currently part of. Along with people, stepping out to explore and create new innovation frames can also be challenging for design practitioners who are engaged and immersed in the existing paradigm (Norman and Verganti, 2014). Designers too have norms and values that act as pre-conceptions that can hinder the innovation process and create resistance to change (Öberg \& Verganti, 2014). Despite this vulnerability, companies still prefer to pursue HCD because it is much easier for incremental innovations to be adopted as the intention of the innovation is to iteratively build on existing frameworks, functionality, and meanings; thereby reducing market uncertainty. Since the HCD approach leverages existing infrastructure and resources, it is easier for companies to innovate rapidly and to continuously improve their product or service offering.

\subsection{Symbiosis as a strategy for mitigating company adoption barriers of D-DI}

As most companies are engaged in HCD, they will now and then need D-DI to remain competitive and profitable. D-DI however, comes with uncertainties and adoption risks. Companies are hesitant to engage with D-DI, but will only do so once they are confident of overcoming all the uncertainties and risks. The HCD approach plays a crucial role in mitigating some or most of these risks. Through its conventional use, HCD can be used to rapidly prototype, test, and validate innovation ideas with people to make sure they are acceptable and usable. However, HCD can also be used unconventionally, most notably to help create a radical new vision in the D-DI process (Verganti, 2016). Insights from the HCD methods like context analysis and people research can be used to create a radical vision which still conforms to desirability, feasibility, and viability, while leveraging existing infrastructure and market desire or appreciation. Used in this way, HCD becomes both a source of validation and inspiration in the D-DI process. HCD here does not necessarily derive the innovation process but rather supports it. While Norman and Verganti propose HCD to follow D-DI, we see the potential for HCD to also precede D-DI.

\section{RESEARCH DESIGN AND METHODOLOGY}

\subsection{Research context and participants}

The context for the research was a six-month graduation project of the second author in fulfillment of a Master of Science degree in Design for Interaction. The project was a collaboration between Delft University of Technology and a major airline company. The airline company, renowned for its excellent customer experience, was keen to explore ways by which the flight disruption experience could be improved in order to ease passenger distress during such disruptions. Seeking to go beyond conventional solutions, the airline company engaged with the second author and the university as external collaborators who suggested coming up with a radical solution that can potentially 'disrupt' flight disruptions. While a D-DI was the goal or desired outcome, the company did not want to deviate from its established work culture and way of working rooted in HCD. Hence, a HCD approach was incorporated in the innovation process as well.

The project involved a radical circle (Verganti, 2016) of six mixed members from the university as well as the airline company: (1) The MSc student with six years of work experience and interest in radical innovation (second author, henceforth 'external designer'); (2) A design practitioner, researcher, and educator as the university project mentor with ten years of work experience and specialization in D-DI (first author); (3) A design researcher and educator as the university project chair with eight years of work experience and specialization in marketing communication (third author); (4) A senior service design strategist from the customer service department with four years of work experience; (5) A project manager and director of the customer service department with sixteen 
years of work experience; and (6) A customer journey manager with eight years of work experience, all as company mentors from the airline company as client.

\subsection{Research approach}

An instrumental design case study was applied (Creswell, 2012) with Research through Design (RtD) as the underlying paradigm (Isley \& Rider, 2018). Reflective practice was deployed for knowledge creation based on design action and reflection in and on action (Schön, 1983). In contrast to D-DI case studies that are often either post-hoc or mere post-it design, RtD inherently affords designerresearchers to project their vision through actual design (Dow et al., 2013). Moreover, the act of designing is where knowledge is generated and design outcomes are considered the material with which the designer-researcher advances an investigation. Subscribing to Boon et al. (2020) who recognize a plurality of RtD styles we practiced our own RtD style.

Data was captured in the form of design documentation (i.a. customer journeys, observations, interviews, surveys, and service blueprints) and written reflections in a journal to act as evidence for debate, constructive assessment, and criticism. The external designer would meet with the university mentors once every week to co-reflect on the project progress and outcomes (Yukawa, 2016). Similar meetings and discussions would take place twice a week with all company stakeholders involved in the project. Over the course of the project, combined meetings with all stakeholders (external and internal teams) were initiated to ensure collective agreement on project goals and progress all the way up to envisioning, prototyping, and implementation. After the project was completed, the external designer and university mentor (both authors of this article) spent several co-reflection sessions analyzing the design documentation and the reflective journal content.

\section{SUBMISSION OF MANUSCRIPT}

\subsection{The design-driven service innovation vision}

The company's customer service meaning was radically innovated from 'reactive operations' to 'proactive empathy'. Having thoroughly explored the flight disruption phenomenon, the external designer was able to highlight the importance of information provided to the passengers during a disruption as a key factor. A new information creation and dissemination framework was designed to promote a proactive interaction with customers. This framework emphasized a more personalized, heartfelt, and sincere feed-forward information experience (Wensveen et al., 2004).

Upon analysis, the design-driven service innovation vision satisfies Dahlin and Behrens' (2005) three criteria for qualifying as a radical innovation (see Section 2). The service was considered novel and unique as no other comparable framework existed within the company's portfolio and was dissimilar to any existing service within the company (Criteria 1 and 2). In fact, the design-driven service innovation vision is currently under a non-disclosure agreement (NDA). The design-driven service innovation vision also has huge potential for building and adding future product and/or service solutions, thereby satisfying the third criteria. Although Dahlin and Behrens' criteria are mostly focused on products being novel, unique, and adopted by the market, Sanberg and Aarikka-stenroos (2014), found newness to the firm also as a criteria sufficient for radical innovation.

The project yielded positive results since the design-driven service innovation vision was adopted by the airline and received as desirable by a test sample of passengers $(\mathrm{N}=50)$. The design-driven service innovation vision is currently planned for implementation. Furthermore, the effectiveness of the new customer relation service demonstrated a notable change in the passengers' perception about the airline's disruption handling experience. In the following section, we explain how the symbiosis of DDI and HCD enabled the airline company to achieve and adopt the design-driven service innovation vision, adding immense value to their current customer service experience.

\subsection{Analysis of the design-driven service innovation vision journey}

Disruption handling experience was not a new problem but rather a recognized issue that the customer experience department of the airline was trying to resolve for a long time. Although equipped with an inhouse design team, attempts to iteratively improve the customer service experience during flight disruptions using a HCD approach were not working as effectively. For example, the self-service kiosks in use for several years were iteratively updated for intuitive usability and line avoidance. Yet, 
the Net Promoter Score (NPS) ratings and passenger survey feedback did not see any significant improvement. Entering the project with this history, the company was curious about D-DI but not open to its risks. Therefore, applying D-DI in symbiosis with HCD, HCD was the approach that the external designer started with. The symbiotic innovation journey in the project consisted of four major phases with related HCD and D-DI activities (see Figure 1): (1) Onboard, (2) Research, (3) Envision, and (4) Implement. The D-DI adoption risk mitigation of each phase is discussed below.

\subsubsection{Phase 1: Onboard}

At the outset of the project, the external designer was introduced to the company. He began to get familiarized with the internal teams and their ways of working by interviewing experts and studying various company documents. In this phase, the project was framed and scoped through multiple discussions with project stakeholders. The airline company saw merit in collaborating with an external designer and the university supervisors as this collaboration created space for a radical exploration as a potential solution to their problem. Having an external designer with a D-DI mindset was seen as a medium to gain new perspectives and go beyond the HCD dominant company culture that was prevalent.

\subsubsection{Phase 2: Research}

In order to get a holistic understanding of the disruptions challenge, the external designer undertook a series of research activities mostly with an HCD approach. As an external designer, it can often be a challenge to acculturate the teams within a company so it is important to build rapport and trust for DDI. The research activities were done with the express aim to gather qualitative and quantitative knowledge, to understand the industry as well as to gain trust within the company. Some of the research activities undertaken were: informal passenger interviews $(\mathrm{N}=5)$; Passenger feedback survey analysis $(\mathrm{N}=2500)$; Passenger conversations over social media analysis $(\mathrm{N}=50)$; Literature review on airline disruption impact and mitigation; Passenger and ground staff interaction observations (Context analysis); Experiencing a mass disruption day first hand; Constructing passenger journey maps; And exploring the current service meaning. Additionally, being external, allowed the external designer to gain distance and reduced the potential for expertise bias, which is normally the case with individuals heavily entrenched in company culture (Taylor, 2018). These research activities also allowed the external designer to understand and gauge the efficacy of existing company infrastructure to increase the chance of the innovation to be adopted, hence implemented.

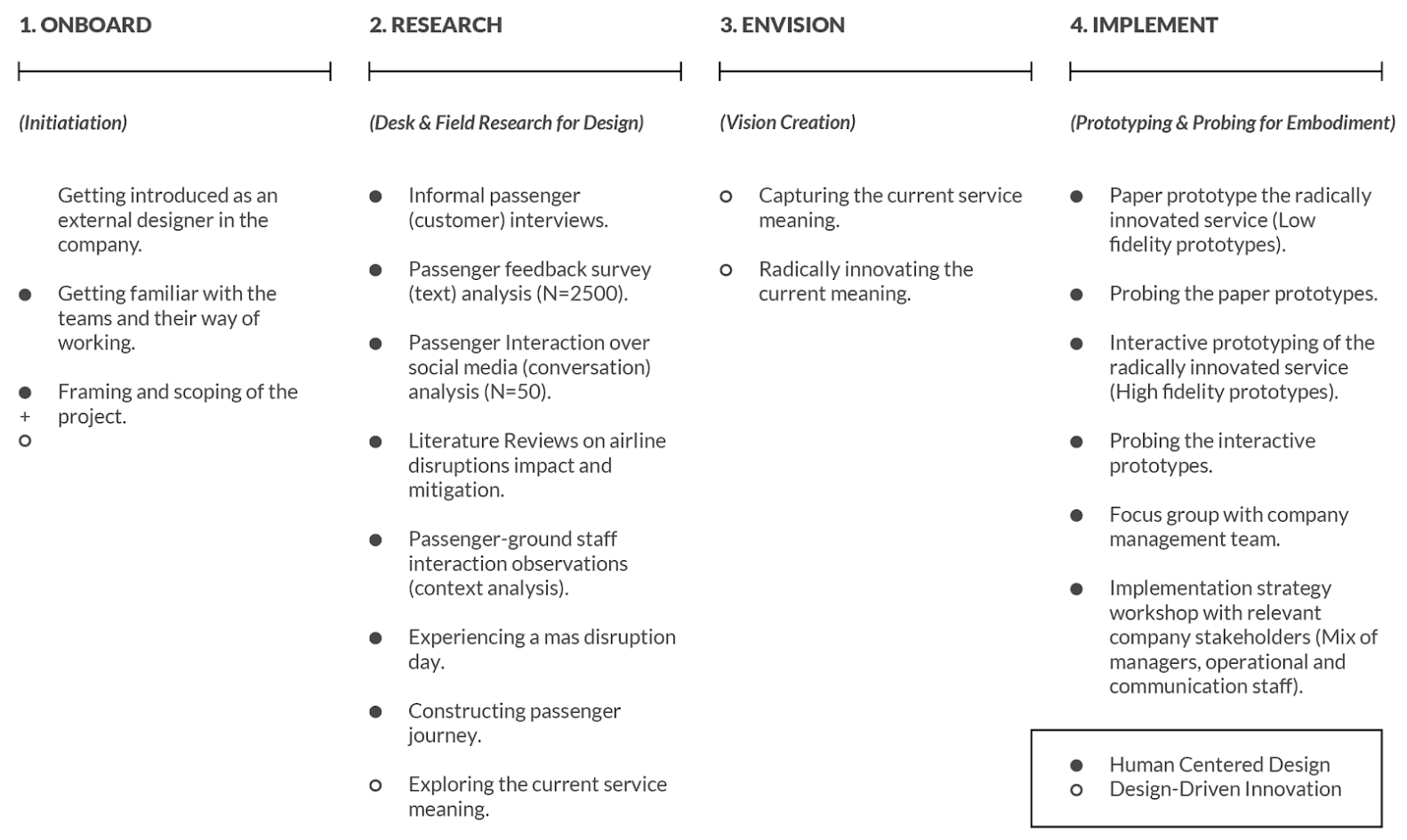

Figure 1: The project process overview with all phases and related $H C D$ and $D-D I$ activities. 


\subsubsection{Phase 3: Envision}

In this phase of the innovation journey, the external designer was able to step back from HCD based insights by not only capturing the initial service meaning, but proposing a radical innovation of the initial service meaning. Stepping back became possible through a deliberate intention to create a D-DI vision and continuous stimulation thereof by university mentors. The D-DI vision resulted from HCD insights inquired beyond the problem context. By not only considering the service front office experience, but considering the service back-office and underlying company perception of the problem as well, the designer was able to thoroughly understand the company's misconception of disruption as a problem. The new proposed experience vision was easily accepted by the company since they were motivated by a solid body of inquiry and analyzed data as evidence. The D-DI was not perceived as too radical for implementation as the constraints of the existing company infrastructure and culture were kept in mind in the innovation process.

\subsubsection{Phase 4: Implement}

In this last phase of the innovation process, the external designer translated the design-driven service innovation vision into solution prototypes. Each prototyping activity was followed up with extensive probes and testing with passengers to gain feedback and refine the radical service innovation. Prototyping activities included paper prototyping the design-driven service innovation vision (low fidelity prototypes) all the way to creating interactive prototypes of the new service (high fidelity prototypes). Tasked with the mandate to also realize the design-driven service innovation vision, the external designer used HCD techniques to conduct focus group sessions and dialogues with the company management teams as well as implementation strategy workshops with all relevant stakeholders (mix of managers, operational, and communication staff).

\section{DISCUSSION AND FINAL WORDS}

Companies need both D-DI and HCD. However, not all companies are open to pursue D-DI (Morillo, et al., 2015). Even when companies are open, they are challenged by traditional ways of working, lack of resources, and infrastructure towards seeking D-DI (Trantow et al., 2011; Von Stamm, 1998). We have explored and analyzed how a design-driven service innovation vision can be instigated and adopted in a company successfully through a symbiosis of D-DI and HCD. As such, this research has three main contributions.

First, considering the hesitance of companies towards D-DI, apart from the critique principle introduced by Verganti (2016), we introduce HCD. Preceding critique, we believe HCD is another strong yet overlooked source for D-DI risk and uncertainty mitigation towards better adoption. The precedent presented in this paper, shows how external designers can successfully collaborate with a company mainly focused on HCD and solve a problem that requires a radical innovation of meaning (a new paradigm) through D-DI. The importance of using a HCD perspective to augment the D-DI process can be highlighted where the external designer gained trust and rapport within the company; thereby enlarging the capacity for D-DI to happen. The HCD approach yielded significant evidence that was crucial for convincing the company about the viability of the design-driven service innovation vision. Along with evidence, it was equally essential to keep in mind the constraints of the existing company infrastructure which made it easier for the innovation to be adopted and implemented. Our approach aligns with Miller et al. (2005) observation regarding the crucial role of obtaining information and processing it into knowledge that can be disseminated and acted upon for accelerated radical innovation to become a commonplace reality. Furthermore, the insights produced through this case study are valuable for building better relationships between design and management which is raised as a concern by Ando and Yaegashi (2017).

Second, although Norman and Verganti (2014) define 'hill finding' through D-DI and 'hill climbing' through HCD as two consecutive categories of innovation, we see the emergence of a new hybrid category - 'finding a new hill on the current hill'. The symbiosis of D-DI and HCD leads to the integration of radical and incremental innovation which we see adoption wise in line with the perspectives of Baha et al. (2012).

Third, we would like to emphasize the importance of external designers for enabling and envisioning D-DI within organizations. The symbiotic use of HCD and D-DI was preceded by the presence of the external designer and the role he played in this innovation journey. Being an external entity allowed the 
designer a certain level of autonomy necessary for bringing a fresh perspective into the company. This autonomy gave the external designer an objectivity with which he observed and critiqued the problem space and the company ethos. However, this critique also generated some tensions between the external designer and the company. For example, the company preferred validation through quantitative data for reaching important milestone decisions, while the designer saw merit in pursuing insights from qualitative data. On top of that, the external designer also had to be cautious not to be influenced or swayed by the dominant HCD culture of the company and influence of the management. The latter is also considered as an area of concern by Ando and Yaegashi (2017). He had to carefully consider when to step in and out of the two approaches. Although external designers are not as experienced about the company culture and operating industry as internal experts, their unfamiliarity can function as a source of innovation depending on their perception, critique, and the ability to radically re-frame. Innovation requires a beginners mindset - "people who are free from pre-conceptions" (Öberg \& Verganti, 2014, p. 206). Aligned with Morillo et al. (2015) who emphasize organization flexibility as giving more control to the interpreter when collaborating with 'outsider' partners, we believe external designers should be given more autonomy and get more attention in D-DI.

The implications of this research also come with limitations. Although an instrumental design case study is a useful first design precedent, more case studies within a varied typology of companies and related products and services are needed to grasp the premise of our first contribution. Next, stressing the ability of the external designer to know when to step in and out during this symbiotic process, we believe this navigation can be challenging for external designers only experienced in one of the two innovation approaches. We therefore recommend either a collaboration between complementary external designers or external designers to simultaneously develop expertise in both approaches. In our case the external designer was a graduate student with expertise in HCD supported by an experienced mentor in D-DI. The mentor could however often only react, not actively act. For future studies it is therefore worth considering a RtD with experienced design experts.

\section{ACKNOWLEDGMENTS}

We would like to thank all the project participants and stakeholders for their support and belief in this project. We also would like to thank the ICED 2021 reviewers for their constructive feedback that helped us to improve the quality of this paper.

\section{REFERENCES}

Ando, T., \& Yaegashi, K. (2017), “Dual Nature of Designer's Attitudes Toward Design-Led Innovation”, The Academy for Design Innovation Management 2017, Ritsumeikan University, Design Research Society, London, pp. 107-125.

Baha, S. E., Lu, Y., Brombacher, A., \& van Mensvoort, K. (2012), "Most Advanced Yet Acceptable, but don't forget”, NordDesign 2012, 22-24 August 2012, Aalborg University, Alborg, pp. 51-58.

Boon, B., Baha, S. E., Singh, A., Wegener, F. E., Rozendaal, M. C., \& Stappers, P. J. (2020), “Grappling with Diversity in Research Through Design”, Design Research Society 2020, 11-14 August 2020, Design Research Society, London, pp. 139-151. https://doi.org/10.21606/drs.2020.362

Brown, T. (2008), "Design Thinking”, Harvard Business Review, pp. 1-10. https://hbr.org/2008/06/design-thinking

Brown, T. (2009), Change by Design: How Design Thinking Transforms Organizations and Inspires Innovation, Harper Collins, New York.

Creswell, J. W. (2012), Educational research Planning, conducting, and evaluating quantitative and qualitative research (4th ed.), Pearson, London, United Kingdom.

Dahlin, K. B., \& Behrens, D. M. (2005), "When is an invention really radical?: Defining and measuring technological radicalness”, Research Policy, Vol. 34 No. 5, pp. 717-737. https://doi.org/10.1016/j.respol.2005.03.009

De Goey, H., Hilletofth, P., \& Eriksson, D. (2016), "Enablers and barriers to design-driven innovation: a case study at a Swedish wood furniture wholesaler”, International Annual EurOMA 2016, 17-22 June 2016, Norwegian University of Science and Technology, Trondheim, pp. 721-743. https://doi.org/10.1108/EBR07-2018-0122

Dell'Era, C., \& Verganti, R. (2007), "Strategies of innovation and imitation of product languages", Journal of Product Innovation Management, Vol. 24 No. 6, pp. 580-599. https://doi.org/10.1111/j.15405885.2007.00273.x

Dooley, L., \& O’Sullivan, D. (2001), “ Structuring innovation: a conceptual model and implementation methodology”, Enterprise and Innovation Management Studies, Vol. 2 No. 3, pp. 177-194.

https://doi.org/10.1080/14632440110101246 
Dow, S., Ju, W., \& Mackay, W. (2009), "Projection, Place and Point-of-view in Research through Design", In Price, S., Jewitt, C., \& Brown, B. (Eds.), The SAGE Handbook of Digital Technology Research, SAGE Publications Ltd., London, pp. 266-285. https://doi.org/10.4135/9781446282229.n19

Fichman, R. G. (2000), “The Diffusion and Assimilation of Information Technology Innovations”, In Zmud, R. W. (Ed.), Framing the domains of IT management: Projecting the future through the past, Pinnaflex Educational Resources inc, Cincinnati, pp. 105-128.

Isley, C. G., \& Rider, T. (2018), "Research-Through-Design: Exploring a design-based research paradigm through its ontology, epistemology, and methodology". Design Research Society 2018, 25-28 June 2018, Design Research Society, London, pp. 357-367. https://doi.org/10.21606/dma.2018.263

Journal of Computer-Supported Collaborative Learning, Vol. 1 No. 2, pp. 203-228. https://doi.org/10.1007/s11412-006-8994-9

Kim, W. C., \& Mauborgne, R. (2005), "Blue ocean strategy: from theory to practice”, California Management Review, Vol. 47 pp. 3, pp. 105-121. https://doi.org/10.1177/000812560504700301

Lai, V. S., Guynes, J. L., \& Bordoloi, B. (1993), "ISDN: Adoption and diffusion issues”, Information Systems Management, Vol. 10 No.4, pp. 46-54. https://doi.org/10.1080/10580539308906957

Miller, L., Miller, R., \& Dismukes, J. (2005), "The critical role of information and information technology in future accelerated radical innovation”, Information Knowledge Systems Management, Vol. 5 No. 2, pp. 63-99.

Liedtka, J., \& Ogilvie, T. (2011), Designing for growth: A design thinking tool kit for managers, Columbia University Press, New York. https://doi.org/10.7312/lied16467

Morillo, M., Dell'Era, C., \& Verganti, R. (2015), "Exploring the role of outsider interpreters in the development of design-driven innovations", International Journal of Technology Intelligence and Planning, Vol. 10 No. 3-4, pp. 222-253. https://doi.org/10.1504/IJTIP.2015.070849

Norman, D. A., \& Verganti, R. (2014), "Incremental and radical innovation: Design research vs. technology and meaning change”, Design Issues, Vol. 30 No. 1, pp. 78-96. https://doi.org/10.1162/DESI_a_00250

Öberg, Å., \& Verganti, R. (2014), "Pre-emptying and the myth of the naïve mind", Academic Design Management 2014, 2-4 September 2014, The Design Management Institute, Boston, 2-4 September 2014.

Rogers, E. M. (1995), "Diffusion of Innovations: modifications of a model for telecommunications", In: Stoetzer, M. W., \& Mahler, A. (Eds.), Die diffusion von innovationen in der telekommunikation. Springer, Berlin, pp. 25-38. https://doi.org/10.1007/978-3-642-79868-9_2

Sandberg, B., \& Aarikka-Stenroos, L. (2014), "What makes it so difficult? A systematic review on barriers to radical innovation”, Industrial Marketing Management, Vol. 43 No. 8, pp. 1293-1305. https://doi.org/10.1016/j.indmarman.2014.08.003

Schön, D. A. (1983), The reflective practitioner: how professionals think in action, Basic Books, New York.

Singh, V. (2013), "Innovation diffusion categories and innovation-related needs", International Conference on Engineering Design 2013, Sungkyunkwan University, 19-22 August 2013, The Design Society, Glasgow. pp. 297-306. https://www.designsociety.org/publication/34925/Innovation+diffusion+categories+and+innovationrelated+needs

Taylor, B. (2018), “The Best Leaders See Things That Others Don’t. Art Can Help”, Harvard Business Review. https://hbr.org/2018/04/the-best-leaders-see-things-that-others-dont-art-can-help

Trantow, S., Hees, F., \& Jeschke, S. (2013), "Innovative Capability - an Introduction”, In: Jeschke, S., Isenhardt, I., Hees, F., \& Henning, K. (Eds.), Automation, Communication and Cybernetics in Science and Engineering 2011/2012, Springer, Berlin, pp. 191-203. https://doi.org/10.1007/978-3-642-33389-7_15

Verganti, R. (2008), "Design, meanings, and radical innovation: A metamodel and a research agenda", Journal of Product Innovation Management, Vol. 25 No. 5, pp. 436-456. https://doi.org/10.1111/j.1540-

5885.2008.00313.x

Verganti, R. (2009), Design driven innovation: changing the rules of competition by radically innovating what things mean, Harvard Business Press, Brighton MA.

Verganti, R. (2011), "Radical design and technology epiphanies: A new focus for research on design management", Journal of Product Innovation Management, Vol. 28 No. 3, pp. 384-388. https://doi.org/10.1111/j.1540-5885.2011.00807.x

Verganti, R. (2016), Overcrowded: Designing Meaningful Products in a World Awash with Ideas. The MIT Press, Cambridge, MA. https://doi.org/10.7551/mitpress/9780262035361.001.0001

Von Stamm, B. (1998), "Whose design is it? The use of external designers", The design journal, Vol. 1 No. 1, pp. 41-53. https://doi.org/10.2752/146069298790225235

Wensveen, S., Djajadiningrat, J. P., \& Overbeeke, C. J. (2004), "Interaction frogger: a design framework to couple action and function through feedback and feedforward", Designing Interactive Systems 2004, Cambridge, Association for Computing Machinery, New York, pp. 177-184. https://doi.org/10.1145/1013115.1013140

Yukawa, J. (2006), "Co-reflection in online learning: Collaborative critical thinking as narrative", International Journal of Computer-Supported Collaborative Learning, Vol. 1 No. 2, pp. 203-228.

https://doi.org/10.1007/s11412-006-8994-9 


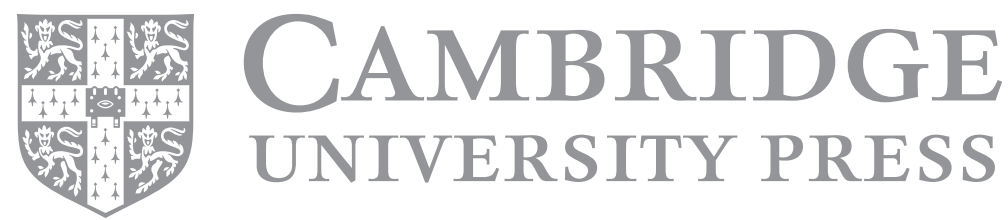

